\title{
Arthroscopy of the knee after the injection of ${ }^{90} Y$
}

\author{
D. B. YATES \\ Charing Cross Hospital, London
}

The technique of internal inspection of the knee by arthroscopy was first described in 1918 by Prof. Takagi of Tokyo, who used a cystoscope to inspect cadaveric knee joints (Watanabe, Takeda, and Ikeuchi, 1969). With the aid of a modern fibreoptic arthroscope, a study is being made of the effect of radioactive yttrium silicate on chronic rheumatoid synovitis of the knee with effusion as part of a longterm study of intra-articular radio-isotope therapy which is in progress at Charing Cross Hospital.

\section{Material and methods}

The arthroscope (Stortz 7200) consists of a light source, introducer, $30^{\circ}$ oblique-viewing telescope, and a smaller end-viewing telescope used in conjunction with biopsy forceps. Arthroscopy has been performed under local anaesthesia with full sterile precautions in the operating theatre. With the knee flexed to $140^{\circ}$ and supported in this position, the arthroscope is inserted via a lateral infrapatellar approach, the joint is visualized, and a biopsy taken. This approach allows good visualization of the

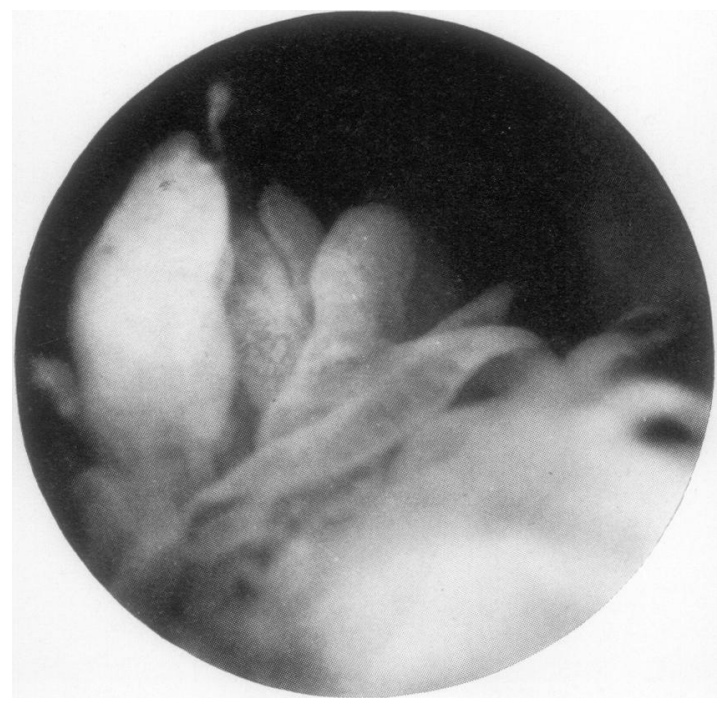

FIG. 1 Rheumatoid synovitis of the knee.Large hyperaemic villi on the medial capsular wall. medial femoral condule, the medial capsular wall, the back of the patella, and the intercondylar notch. Biopsy specimens have been taken from a constant area, high on the medial capsular wall.

From studies in experimental arthritis (Glynn, 1972) and early joint lesions in rheumatoid arthritis (Kulka, Bocking, Ropes, and Bauer, 1955), a progression of changes in the rheumatoid synovial membrane is found. The normal joint appears as cream-coloured smooth articular cartilage with pale pink, even surfaced, synovium. In the first stage of rheumatoid arthritis, the synovial membrane shows conspicuous surface blood vessels and small oedematous villi. Histologically, the synovium at this stage shows a moderate increase in vascularity with a slight diffuse mononuclear cell infiltration.

In the next stage, the villi are more prominent and polypoidal-with a waxy-looking surface which also contains prominent blood vessels. In histological section, these villi contain many blood vessels, and a now intense mononuclear response. In the third stage, the increase in synovial tissue caused by villous proliferation leads to partial overgrowth of the articular cartilage by synovium so-called pannus formation-, and this in turn leads to destruction of the cartilage and erosion.

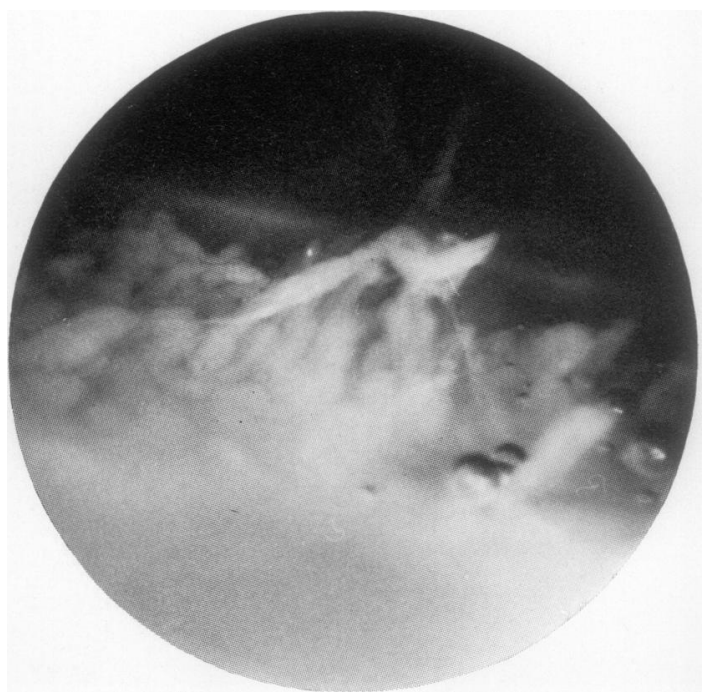

FIG. 2 Same area as in Fig. 1 one month after injection of ${ }^{90} Y$. Villi now less prominent and avascular. 
The final stage of activity, coupled with some villous proliferation, and usually seen in joints with long-standing rheumatoid arthritis, is that of fibrin production. This occurs either as synovial surface deposition, appearing as opaque white avascular villi, or as loose threads or aggregates, free within the joint cavity-the so-called 'rice bodies'. In both cases, the histology shows a mass of eosinophilic material containing few cells, and probably represents the final stage of an inflammatory response.

Arthroscopy and synovial biopsy have been performed 10 days before, and 4 weeks after, the intra-articular injection of $5 \mathrm{mCi} .{ }^{90} \mathrm{Y}$ silicate. After injection, a backslab splint was applied to the treated knee and weight-bearing was forbidden for 6 days. To avoid error due to sample variation. an area of synovium high on the medial capsular wall was specifically chosen for photography and biopsy. The results from two patients are shown.

\section{Results}

The most obvious change seen after ${ }^{90} \mathrm{Y}$ is a marked depletion in the number of synovial villi. Those villi that are present are less hyperaemic and appear to be opaque white masses of fibrinous material. There is also a moderate increase in the amount of the loose fibrin seen floating in the joint cavity (Figs 1 and 2).

Biopsy specimens were examined for features consistent with rheumatoid synovitis according to the 1958 criteria of the American Rheumatism Association (Ropes, Bennett, Cobb, Jacox, and Jessar, 1959). These consist of mononuclear cell infiltration, surface fibrin deposition, perivascular infiltration, perivascular oedema, and increased lining layer thickness.

It can be seen that after ${ }^{90} \mathrm{Y}$, there is a considerable increase in synovial lining layer thickness with an increase in fibrin deposition in this layer (Figs 3 and 4, overleaf). There is no appreciable change in deeper synovial structures and no change in mononuclear cell infiltration. It has been postulated that, in rheumatoid synovitis, the greater the mononuclear cell infiltration (particularly lymphocytic) and the thinner the layer of lining cells, the lesser is the underlying cartilaginous and bony destruction (Muirden and Mills, 1971). The changes seen after ${ }^{90} \mathrm{Y}$ may, however, be the result of a temporary reactive synovitis arising from exposure to local radioactivity.

In summary, therefore, intra-articular ${ }^{90} \mathrm{Y}$ leads to changes in the arthroscopic appearance of the synovium, and also to changes in synovial histology. These suggest that the early effect of the treatment is directed, as might be expected, against the superficial layer of the synovium.

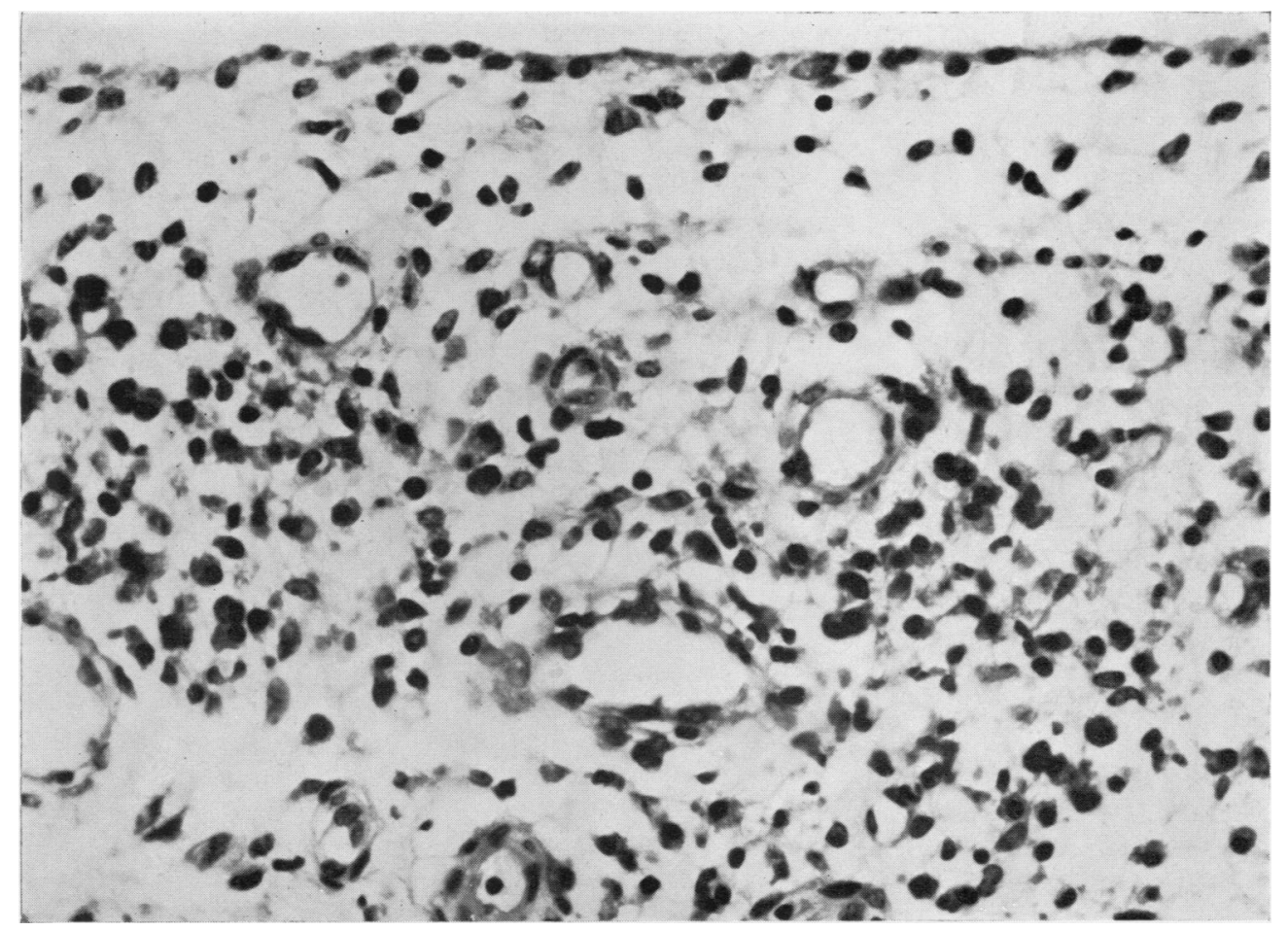

FIG. 3 Rheumatoid synovitis before treatment. $\times 400$ 


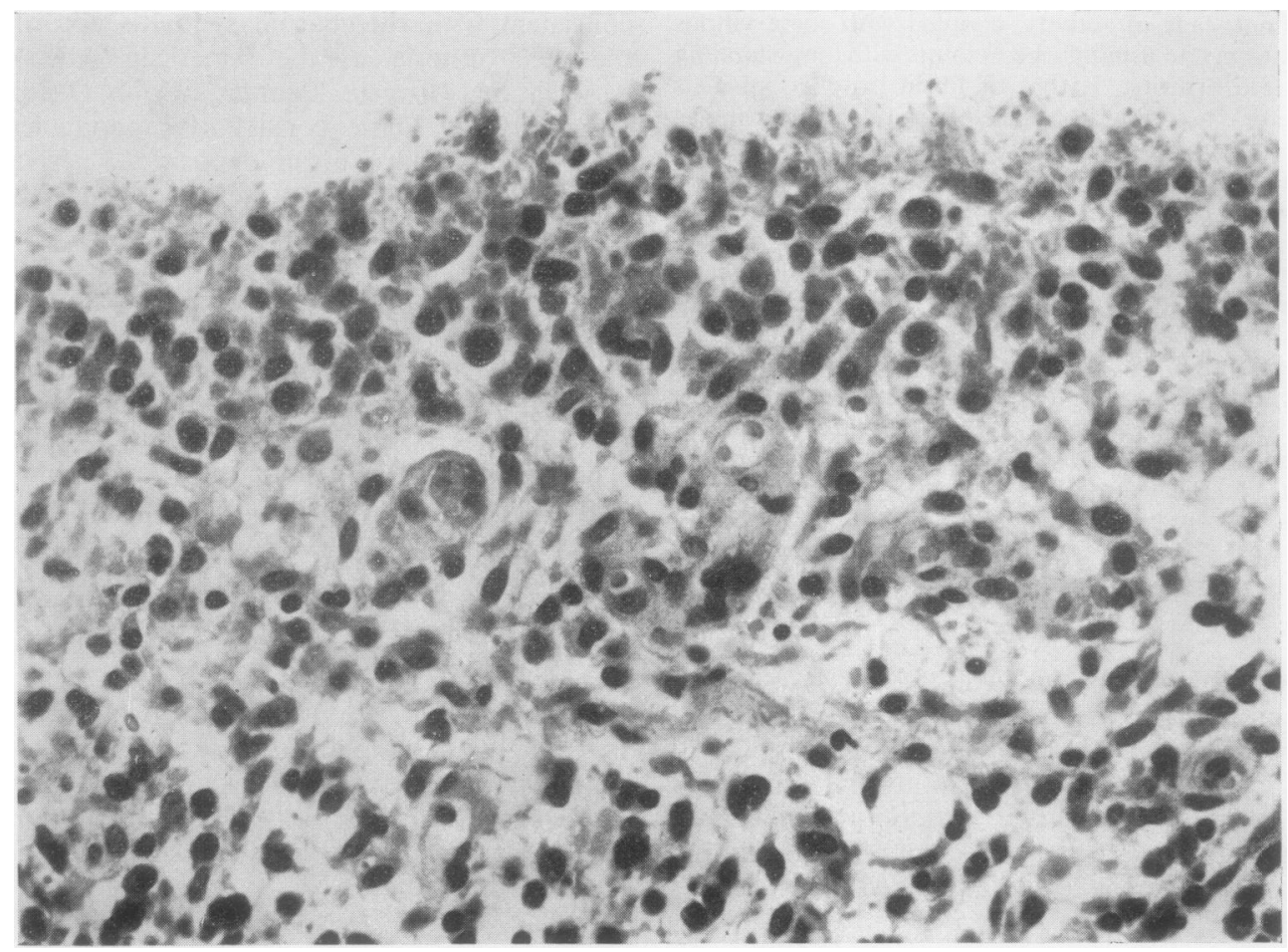

FIG. 4 Synovium one month after injection of ${ }^{90} Y$. Increased lining layer thickness with no change in intensity of mononuclear cell infiltration. $\times 400$ 